Running head: OBSERVED SCORE RELIABILITY IN DCMS

\title{
Observed Score Reliability Indices in Diagnostic Classification Models
}

This paper is accepted to Behaviormetrika and its DOI is 10.1007/s41237-021-00153-9.

\author{
Kazuhiro Yamaguchi \\ University of Tsukuba \\ Jonathan Templin \\ The University of Iowa
}

\begin{abstract}
Author Note
Kazuhiro Yamaguchi (D) https://orcid.org/0000-0001-8011-8575

Jonathan Templin (D) https://orcid.org/0000-0001-7616-0973

On behalf of all authors, the corresponding author states that there is no conflict of interest. This work was supported by JSPS Grant-in-Aid for JSPS Research Fellow $18 J 01312$ and JSPS KAKANHI 19H00616, 20H01720, $21 \mathrm{H} 00936$.

Correspondence concerning this article should addressed to Kazuhiro Yamaguchi, Faculty of Human Sciences, University of Tsukuba, A314 Institutes of Human Sciences A, 1-1-1 Tennodai, Tsukuba-shi, Ibaraki-ken 305-0006, Japan.

Email: yamaguchi.kazuhir.ft@u.tsukuba.ac.jp
\end{abstract}




\title{
Observed Score Reliability Indices in Diagnostic Classification Models
}

\begin{abstract}
Quantifying the reliability of latent variable estimates in diagnostic classification models has been a difficult topic, complicated by the classification-based nature of these models. In this study, we derive observed score reliability indices based on diagnostic classification models as an extension of classical test theory-based reliability. Additionally, we derive conditional observed sum- and sub-score distributions. In this manner, various conditional expectations and conditional standard error of measurement estimates can be calculated for both sum- and sub-scores of a test. The proposed methods provide a variety of expectations and standard errors for attribute estimates, which we demonstrate with an analysis of an empirical test. Moreover, a simulation study revealed the proposed sub-score based reliability index was correlated to a previously developed attribute mastery reliability index.
\end{abstract}

Keywords: diagnostic classification models, cognitive diagnostic models, sum- and subscore analyses, conditional standard errors, reliability index 


\section{Observed Score Reliability Indices in Diagnostic Classification Models}

As their name connotes, diagnostic classification models (DCMs; e.g., Rupp, Templin, \& Henson, 2010), also known as cognitive diagnosis models (CDMs; e.g., Leighton \& Gierl, 2007), are classification-based models where examinees are put into classes purporting to represent their knowledge state across multiple latent attributes. Having latent attributes that are classificationbased, however, frequently puts DCMs at odds with psychometric methods and modeling paradigms that seek to place examinees onto a common scale, such as classical test theory (CTT) or item response theory (IRT). To quantify reliability in a more uniform manner with scale-based psychometric methods, we propose the use of observed score reliability indices under DCMs to evaluate how raw scores from DCM-based assessments represent the latent attributes for gaining clues to improve diagnoses. Moreover, to define raw score-based reliability indices, conditional expectations and standard errors of measurement of raw scores in DCMs (which are classically derived psychometric statistics) are also derived.

DCMs can provide a profile of strengths and weaknesses of examinees' cognitive abilities. In DCMs, these multiple latent abilities are often binary, representing mastery or nonmastery of the cognitive elements underlying a test. A specific permutation of attribute mastery statuses is called an attribute mastery pattern. A number of DCMs have been developed to represent various assumptions linking attributes and item responses (e.g., DiBello, Roussos, \& Stout, 2006; Ravand \& Baghaei, 2019; Rupp et al., 2010). Well-known DCMs include the loglinear cognitive diagnosis model (LCDM; Henson, Templin, \& Willse, 2009), generalized deterministic input noisy and gate model (G-DINA; de la Torre, 2011), and general diagnostic model (GDM; von Davier, 2008).

Perhaps the most informative aspect of an analysis with a DCM is the examinee-specific estimate of their attribute mastery pattern and, for each attribute individually, the attribute mastery probability. Therefore, DCM score reports concentrate on estimated attribute patterns (e.g., Liu, Qian, Luo, \& Woo, 2018). Although there are various types of DCM reliability 
indices, a common critique of DCM-based studies is that they often lack reliability reports (Sessoms \& Henson, 2018). As an example of reliability for DCMs, originally, attribute pattern estimation accuracy and consistency were the main definitions of reliability of DCMs (e.g., Cui, Gierl, \& Chang, 2012; Johnson \& Sinharay, 2018; Wang, Song, Chen, Meng, \& Ding, 2015). In addition, Johnson and Sinharay (2019) assumed the posterior probability of attribute mastery patterns as an observed score and defined reliability which was the correlation of the true attribute mastery pattern and the observed score. Templin and Bradshaw (2013) defined of reliability indices based on simulated test-retest classification consistency. Another interesting definition of reliability was based in information theory called mutual information reliability (Chen et al., 2018). Finally, Gierl, Cui, and Zhou (2009) introduced a weighted score-based coefficient similar to the classical alpha coefficient.

Most of these reliability indices were focused on attribute mastery probabilities (for each attribute, individually or marginally) or full attribute mastery patterns. However, these indices do not provide information about how mastery of specific latent attributes affects the raw test score. As such, these previous indices are somewhat removed from the actual tests which serve as the basis for diagnoses. To believe a diagnostic result, we also must evaluate the quality of the test. Moreover, for DCM-based assessment users who are educational practitioners, such as teachers, understandable information about the quality of their tests is important as it can be used to improve these instruments. Test sub-scores have frequently been used to provide diagnostic information of more narrowly-focused content areas (e.g., Sinharay, Puhan, \& Haberman, 2010). For example, Haberman (2008) proposed a new rule to report sub-scores based on CTT and Yao (2010) employed multidimensional item response theory (MIRT) for the same purpose. Many studies have investigated the properties of or how to estimate test sub-scores. Further, many studies have investigated the low reliability of sub-scores and developed methods to improve these estimates (e.g., Feinberg \& Jurich, 2017; Sinharay, 2010).

Classically derived test scores, such as sum scores or sub-scores, are still the basic metric in test data analysis and are meaningful for teachers and students because of their familiarity and 
ease of calculation (Henson, Templin, \& Douglas 2007). Such scores can be used in a DCMbased testing context. For instance, Lai, Gierl, and Babenko (2015) showed a flow of diagnostic assessment and indicated that the diagnostic reporting contained two aspects: probabilistic model scoring and subscale scores. Probabilistic model scoring gives estimates of attribute pattern or attribute mastery probabilities and sub-scale scoring was based on classical sub-scores from a test. Lai et al. (2015) also provided conditional average sub-scores based on the attribute hierarchy model. However, raw scores based such reports have not received much attention in DCM research.

DCMs have allowed us to provide sophisticated information about students' learning statuses without classical sum- and sub-scores, the latter of which may have low reliabilities. However, sum- and sub-scores can reflect the quality of a test. Like CTT and IRT, mastering specific attributes implies higher test scores in DCMs, stemming from DCM parameter monotonicity constraints (Henson, et al. 2009). If test scores do not reflect such differences between attribute mastery and non-mastery, then the test is not appropriate for diagnostic purposes and such a low-quality test will make the estimation of attribute mastery unclear. Thus, we can use test scores to assess the quality of a diagnostic test. Statistically sophisticated methods to check item level discrimination quality exist (e.g., de la Torre, 2008; Henson, DiBello, \& Stout, 2018), but one benefit of sum- and sub-score based test assessment methods is their applicability in many situations because they do not require complex calculations. Moreover, interpretations of the scores are also straight forward and can be performed by classroom teachers or others who are not well-versed in psychometrics.

Henson et al. (2007) noted the utility of the sum- and sub-scores derived under DCM contexts, as such scores are useful to communicate with stakeholders such as teachers and students because they are more familiar with traditional test scores than they are with DCMbased attribute mastery probabilities. Such easy-to-understand scores are also beneficial to test developers. Even in the context of IRT models (which result in continuously-valued estimates of latent ability), the observed score metric is frequently used to communicate scores, with latent 
ability estimates translated onto the observed score metric. Therefore, model implied sum- and sub-scores can provide understandable metrics and clues to improve a test. In the case of use of the observed score, we do want to know the reliability of an attribute-based sum-score. If the reliability is too low, the test score may become a target of development. Finally, test development is never complete; tests need continuous improvement. Sum- and sub-scores provide raw but interpretable perspectives as assessment tools. In summary, we believe focusing on the sum score (or sub-score related metrics) is important and still has value not only in test interpretation but also test development, even if a test was DCM-based.

To define observed score reliability indices under DCMs, we must derive the observed test score distributions that are conditional on marginal and joint attribute pattern mastery statuses. These distributions are not only employed to define reliability but also are informative to understand the test itself. The conditional distribution of a test score given an examinee's attribute mastery pattern reflects the mean and variance of a raw score for an examinee with that pattern. A similar argument was proposed by Liu, Huggins-Manley, and Bulut (2018) who presented the relationship between an estimated unidimensional IRT score and an attribute mastery pattern using box plots to discuss the mastery process of attributes. This approach was one way to understand the relative difficulty of mastering each attribute. Moreover, deriving observed score distributions reveals conditional expectations of sum- and sub-scores in DCMs. This makes it possible to construct assessments with expected the sum- and sub-scores in DCMs in the same way as IRT models use test characteristic curves.

In addition to above metrics, the Standards for educational and psychological testing (AERA, APA, and NCME, 2014, p.39) note that the conditional standard error of measurement (CSEM; e.g., Feldt, Steffen, \& Gupta, 1985) is more informative than the overall standard error of measurement (SEM), which is an overall precision index and does not provide specific scorelevel differences in measurement accuracy. The Standards also suggest that CSEMs should be reported at several score levels (Standard 2.14, p.46). Furthermore, conditional average scores and the attribute-specific precision of scores, which are CSEMs under DCMs, are also important 
in the construction of a test. For example, the attribute mastery pattern conditional raw score provides a measure of how difficult or easy the test was for examinees with that specific attribute pattern. In addition, the precision of the conditional raw score is required to interpret test scores. If a conditional raw score were to have large variability, the test might not appropriately reflect latent attributes alone, having other dimensions that may cause the variability. However, conditional distributions of test scores have not been derived under DCMs.

Our motivation for this study is to provide theoretical support for DCM-based observed score reliability indices to provide additional assessment tools for diagnostic tests. Moreover, we also show observed sum- and sub-score related metrics that are conditional expectations and standard efforts of measurement that utilize the basic test metrics. We believe such indices have value in that they tie the latent variables to the observed score metric - a metric that is easily understood by practitioners and educational measurement researchers. The observed score indices derived in this article can become part of a toolbox to help to assess the nature of a test in an easily interpretable way. In the next section, we introduce general DCMs, define DCM-based observed score reliability indices, and derive DCM-based conditional sum- and sub-scores. We then analyze an empirical data set with these derived conditional distributions and calculate the reliability of such scores. We also conduct a simulation study to compare proposed score reliability and a previously developed attribute level reliability. Finally, we discuss how to use DCM-based observed scores and how these can be incorporated into test construction and analysis processes.

\section{Model Formulation of DCMs and Conditional Observed Scores}

\section{General DCM Formulation}

In general, DCMs are restricted latent class models (Rupp \& Templin, 2008) where latent classes represent attribute mastery patterns, which we represent with the index $c$. Then, $\boldsymbol{\alpha}_{c}=\left(\alpha_{c 1}, \ldots, \alpha_{c a}, \ldots, \alpha_{c A}\right)^{\top}$ is a profile of $A$ latent binary attributes with index $a$, where each element $\alpha_{c a}$ is binary-valued: a one indicates mastery of attribute $a$ and a zero indicates nonmastery. The total number of latent classes/attribute mastery patterns is $2^{A}$. 
Although any DCM can be used for the methods outlined in this article, we focus on the loglinear cognitive diagnosis model (LCDM). To show the LCDM item response function, let $X_{c i}$ be a binary response of an examinee with attribute mastery pattern $c$ to item $i(i=1, \ldots, I)$. Then, the sum score is denoted as $X_{c} .=\sum_{i=1}^{I} X_{c i}$. The item response function of the LCDM for an item $i$ is:

$$
\mathrm{P}\left(X_{c i}=1 \mid \boldsymbol{\alpha}_{c}\right)=\frac{1}{1+\exp \left(-\left(\lambda_{i, 0}+\lambda_{i}^{\top} h\left(\boldsymbol{\alpha}_{c}, \boldsymbol{q}_{i}\right)\right)\right)^{\prime}}
$$

where the kernel function $\boldsymbol{\lambda}_{i}^{\top} h\left(\boldsymbol{\alpha}_{c}, \boldsymbol{q}_{i}\right)$ provides the set of LCDM item parameters (main effects and interactions) and can be represented as:

$$
\boldsymbol{\lambda}_{i}^{\top} h\left(\boldsymbol{\alpha}_{c}, \boldsymbol{q}_{i}\right)=\sum_{a=1}^{A} \lambda_{i, 1,(a)} \alpha_{c a} q_{i a}+\sum_{a=1}^{A-1} \sum_{a^{\prime}>a}^{A} \lambda_{i, 2,\left(a, a^{\prime}\right)} \alpha_{c a} \alpha_{c a^{\prime}} q_{i a} q_{i a^{\prime}}+\cdots
$$

Here, $\boldsymbol{q}_{i}$ is a row vector from the Q-matrix (Tatsuoka, 1983), which indicates the attributes measured by each item. An element of the Q-matrix, $q_{i a}$, takes 1 if attribute $a$ is measured by item $i$ and otherwise is $0 . \lambda_{i, 0}$ is an item intercept parameter and $\lambda_{i}=\left(\lambda_{i, 1,(1)}, \lambda_{i, 1,(2)}, \ldots\right)^{\top}$ is a vector of potential item parameters, realized when corresponding elements of $h\left(\boldsymbol{\alpha}_{c}, \boldsymbol{q}_{i}\right)$, a vector-valued function, are one. $\lambda_{i, 1,(a)}$ is a conditional main effect parameter of attribute $a$ and $\lambda_{i, 2,\left(a, a^{\prime}\right)}$ is a two-way interaction parameter between attributes $a$ and $a^{\prime}$. For the simplicity we denote $\mathrm{P}\left(X_{c i}=1 \mid \boldsymbol{\alpha}_{c}\right)=\omega_{c i}$ as the probability of a correct response for a person with latent class/attribute profile $c$ to item $i$. The set of attribute profiles $\boldsymbol{\alpha}$ are assumed to follow a multivariate Bernoulli distribution with parameters that represent the probability an examinee has a given latent class/attribute profile $\pi=\left(\pi_{1}, \ldots, \pi_{c}, \ldots, \pi_{2} A\right)^{\top}$, with $\sum_{c=1}^{2^{A}} \pi_{c}=1$.

\section{Conditional Sum- and Sub-scores of the DCMs}

For an analysis with a DCM, if the data fit the model, conditional independence of item responses given a latent class/attribute mastery pattern holds. Therefore, conditional item responses follow Bernoulli distributions with probability parameter $\omega_{c i}$, which we represent as 
$X_{c i} \mid \boldsymbol{\alpha}_{c} \sim \operatorname{Bernoulli}\left(\omega_{c i}\right)$. Then, the distribution of the conditional sum score, $X_{c} \cdot \mid \boldsymbol{\alpha}_{c}=$

$\sum_{i=1}^{I} X_{c i} \mid \boldsymbol{\alpha}_{c}$, follows a sum-binomial distribution (described subsequently), which we represent

as $X_{c} \cdot \mid \boldsymbol{\alpha}_{c} \sim \sum_{i=1}^{I} \operatorname{Bernoulli}\left(\omega_{c i}\right)$. In addition, the conditional expectation of the sum score,

which follows a sum-binomial distribution, is $\mathbb{E}\left[X_{c} \mid \boldsymbol{\alpha}_{c}\right]=\sum_{i=1}^{I} \mathbb{E}\left[X_{c i} \mid \boldsymbol{\alpha}_{c}\right]=\sum_{i=1}^{I} \omega_{c i}$ and its

conditional variance is $\mathbb{V}\left[X_{c} \cdot \mid \boldsymbol{\alpha}_{c}\right]=\sum_{i=1}^{I} \mathbb{V}\left[X_{c i} \mid \boldsymbol{\alpha}_{c}\right]=\sum_{i=1}^{I} \omega_{c i}\left(1-\omega_{c i}\right)$. The conditional

expectation is the sum of correct item response probabilities of the attribute profile $\boldsymbol{\alpha}_{c}$. In

addition, the variance is the usual sum of variances of each item response of the attribute profile $\boldsymbol{\alpha}_{c}$.

Whereas $X_{c} \cdot \mid \boldsymbol{\alpha}_{c}$ is the conditional test score (conditional on attribute profile $\boldsymbol{\alpha}_{c}$ ), typically, we use a marginal test score $X$. without respect to $\boldsymbol{\alpha}_{c}$. To calculate the marginal test score, we marginalize across the distribution of the latent variables $\boldsymbol{\alpha}$. In a DCM, the marginal test score follows a mixture distribution. Therefore, from general mixture distributions, the expectation and variance of the marginalized test scores are:

$$
\begin{gathered}
\mathbb{E}[X .]=\sum_{c=1}^{2^{A}} \pi_{c}\left(\sum_{i=1}^{I} \omega_{c i}\right) \\
\mathbb{V}[X .]=\sum_{c=1}^{2^{A}} \pi_{c}\left[\left(\sum_{i=1}^{I} \omega_{c i}\right)^{2}+\sum_{i=1}^{I} \omega_{c i}\left(1-\omega_{c i}\right)\right]-\left[\sum_{c=1}^{2^{A}} \pi_{c}\left(\sum_{i=1}^{I} \omega_{c i}\right)\right]^{2} .
\end{gathered}
$$

Equation (3) is weighted sum of the conditional sum score. The first term in Equation (4) is composed from the squared moment of the conditional sum-score and its variance. In addition, the second term is the square of the weighted sum of the conditional sum-score.

We can also create a conditional sum score for mastery of a given attribute $\alpha_{a}$ by marginalizing across all other attributes. The conditional sum score given $\alpha_{a}$ represents the impact of attribute mastery on the score. In other words, if the difference in scores between 
masters and non-masters of an attribute is large, the test score has a high degree of discrimination between the mastery and non-mastery statuses of the attribute. Conversely, if the difference is small, the test score does not distinguish between attribute mastery and non-mastery. This conditional sum score can be gained by using a marginalized mixing probability. We will denote $c^{\prime}$ as an attribute mastery pattern including $\alpha_{a}=1$, The conditional mixing probability is calculated as

$$
\pi_{c^{\prime}}^{\left(\alpha_{a}=1\right)}=\frac{\pi_{c^{\prime}}}{\sum_{\alpha_{c a}=1 \in \alpha_{c}} \pi_{c^{\prime}}}
$$

where summation is taken across attribute mastery patterns where the $a$-th attribute is equal to one. Then, using this probability as mixture weight in Equations 3 and 4 , we modify $\omega_{c i}$ as the conditional correct item response probability given $\alpha_{a}=1$. We then calculate the expectation of the sum score conditional on the $a$-th attribute being mastered $\left(\alpha_{a}=1\right)$. Similarly, we could also do so when the $a$-th attribute is not mastered $\left(\alpha_{a}=0\right)$. In this case, we change the conditional mixing probability in Equation 5 to be taken over all attribute patterns where $\alpha_{a}=$ 0 .

The conditional sub-score of $a$-th attribute is defined as:

$$
X^{(a)}\left|\boldsymbol{\alpha}_{c}=\sum_{i=1}^{I} q_{i a} X_{c i}\right| \boldsymbol{\alpha}_{c}
$$

The $q_{i a}$ terms select items related to the $a$-th attribute based on their respective entries in the Q-matrix. Likewise, $X^{(a)} \mid \boldsymbol{\alpha}_{c}$ follows a sum-binomial distribution. Similar to the total test score, the conditional expectation of the sub-score of the $a$-th attribute is $\mathbb{E}\left[X^{(a)} \mid \boldsymbol{\alpha}_{c}\right]=\sum_{i=1}^{I} q_{i a} \omega_{c i}$ and the conditional variance is $\mathbb{V}\left[X^{(a)} \mid \boldsymbol{\alpha}_{c}\right]=\sum_{i=1}^{I} q_{i a} \omega_{c i}\left(1-\omega_{c i}\right)$. The basic calculation of the 
marginalized $a$-th attribute sub-score is same as the marginalized sum score. Therefore, the expectation and variance of the marginalized $a$-th attribute sub-score are

$$
\begin{gathered}
\mathbb{E}\left[X^{(a)}\right]=\sum_{c=1}^{2^{A}} \pi_{c}\left(\sum_{i=1}^{I} q_{i a} \omega_{c i}\right) \\
\mathbb{V}\left[X^{(a)}\right]=\sum_{c=1}^{2^{A}} \pi_{c}\left[\left(\sum_{i=1}^{I} q_{i a} \omega_{c i}\right)^{2}+\sum_{i=1}^{I} q_{i a} \omega_{c i}\left(1-\omega_{c i}\right)\right]-\left[\sum_{c=1}^{2^{A}} \pi_{c}\left(\sum_{i=1}^{I} q_{i a} \omega_{c i}\right)\right]^{2} .
\end{gathered}
$$

The conditional sub-score given the $a$-th attribute value is calculated the same as total test score. Equations 7 and 8 are parallel to Equation 3 and 4.

To estimate the conditional expectations or variances, maximum likelihood or Bayesian estimates of $\omega_{c i}$ and $\pi_{c}$ can be used. Marginal maximum likelihood estimates can be obtained by Mplus (Templin \& Hoffman, 2013) or some R packages (e.g., de la Torre \& Akbay, 2019; George, Robitzsch, Kiefer, Groß, \& Ünlü, 2016; Ravand, 2015).

\section{Reliability Index Based on the Conditional Score Distributions}

The reliability index under classical test theory assumes independence of true score and random error and is defined as

$$
\rho_{\mathrm{CTT}}=\frac{\text { Variance of true score }}{\text { Variance of observed score }}=1-\frac{\text { Variance of error }}{\text { Variance of observed score }} \text {. }
$$

We borrow this idea to define the reliability of a test score under DCMs which is defined as

$$
\rho_{\mathrm{DCM}}=1-\frac{\sum_{c=1}^{2^{A}} \pi_{c} \mathbb{V}\left[X . \mid \boldsymbol{\alpha}_{c}\right]}{\mathbb{V}[X .]} .
$$

The denominator $\mathbb{V}[X$.$] is total variance of sum scores. The numerator \sum_{c=1}^{2^{A}} \pi_{c} \mathbb{V}\left[X . \mid \boldsymbol{\alpha}_{c}\right]$ is a weighted sum of the conditional variances. The conditional variance $\mathbb{V}\left[X . \mid \boldsymbol{\alpha}_{c}\right]$ can be interpreted as an error variance of the sum score in an attribute mastery pattern $\boldsymbol{\alpha}_{c}$ because the variance cannot be decomposed into finer pieces. In other words, from analysis of variance, 
$\mathbb{V}\left[X . \mid \boldsymbol{\alpha}_{c}\right]$ is the variance of test scores within an attribute mastery pattern. Therefore $\rho_{\mathrm{DCM}}$ gives the proportion of variance due to sum score differences among attribute mastery patterns.

Often in DCMs, monotonicity constraints are used for model identification, such that the conditional item response probability for attribute profiles with additional Q-matrix indicated attributes mastered is monotonically increasing. Therefore, the between-attribute mastery pattern variance indicates how the test can distinguish the difference among attribute mastery patterns.

As in the previous definition, we can define the effect of attributes on sum-scores as

$$
\rho_{\mathrm{DCM}}^{\left(\alpha_{a}\right)}=1-\frac{\mathbb{V}\left[X . \mid \alpha_{a}=1\right]\left(\sum_{\alpha_{a}=1 \in \boldsymbol{\alpha}_{c}} \pi_{c}\right)+\mathbb{V}\left[X . \mid \alpha_{a}=0\right]\left(\sum_{\alpha_{a}=0 \in \boldsymbol{\alpha}_{c}} \pi_{c}\right)}{\mathbb{V}[X .]} .
$$

We can interpret $\rho_{\mathrm{DCM}}^{\left(\alpha_{a}\right)}$ as the amount of variance in the total test score explained by the $a$-th attribute. If this value is small, the attribute does not have any influence on the test score.

In addition, we can define sub-score reliability using the concept of variance decomposition and Equation 8, which is

$$
\rho_{\mathrm{DCM}}^{(a)}=1-\frac{\sum_{c=1}^{2^{A}} \pi_{c} \mathbb{V}\left[X^{(a)} \mid \boldsymbol{\alpha}_{c}\right]}{\mathbb{V}\left[X^{(a)}\right]} .
$$

The $\rho_{\mathrm{DCM}}^{(a)}$ is amount of sub-score variance explained by the attribute mastery pattern where all attributes are mastered. Finally, the sub-score reliability of the $a$-th attribute is represented as

$$
\rho_{\mathrm{DCM}}^{\left(a, \alpha_{a}\right)}=1-\frac{\mathbb{V}\left[X^{(a)} \mid \alpha_{a}=1\right]\left(\sum_{\alpha_{a}=1 \in \boldsymbol{\alpha}_{c}} \pi_{c}\right)+\mathbb{V}\left[X^{(a)} \mid \alpha_{a}=0\right]\left(\sum_{\alpha_{a}=0 \in \boldsymbol{\alpha}_{c}} \pi_{c}\right)}{\mathbb{V}\left[X^{(a)}\right]} .
$$

The proposed observed sum- and sub-score reliabilities are the result of an application of the concepts of CTT-based reliability. The essential point is the ratio of observed score variance explained by the latent attribute or attribute mastery pattern. Therefore, the observed score reliabilities can be interpreted similar to effect sizes from analysis of variance. If the reliabilities are small, attribute mastery has a small effect on sum- or sub-score. These proposed observed score reliability indices provide a relationship between attribute mastery and interpretable sum- and sub-scores. In other words, observed score-based reliabilities provide 
evidence about the attribute itself. Previously proposed reliability referring to correct classification cannot provide such information.

\section{Application to Empirical Data}

\section{Data and Analysis Settings}

To demonstrate the results of the DCM-based sum scores and reliability indices, we use an example data set from the Examination for the Certificate of Proficiency in English (ECPE) (e.g.; Templin \& Bradshaw, 2014; Templin \& Hoffman, 2013). The ECPE had 28 items and a sample of 2922 examinees. The data are available from the R package GDINA (Ma \& de la Torre, 2017). Following the analysis in Templin \& Hoffman (2013), we set the number of attributes to three, representing examinee mastery of (1) morphosyntactic rules, (2) cohesive rules, and (3) lexical rules of the English language. The Q-matrix is shown in Table 1. None of the items measured all three attributes. Nineteen items measured one attribute, and nine items measured two attributes. The first, second, and third attribute were measured by 13, 6, and 18 items, respecively. Estimation of DCM parameters was conducted using Mplus version 8.1 for Windows (Muthén \& Muthén, 1998-2017) although we note that our analysis can be conducted using the estimated parameters presented in Templin and Hoffman (2013).

\section{Results}

Table 2 shows attribute mastery patterns along with $\pi_{c}$ - the estimated proportion of examinees having each attribute pattern. The proportion of examinees mastering all three attributes (pattern 111) had the highest estimated probability (.346) followed by the probability for the pattern with all attributes not mastered $(000 ; .301)$. Pattern 5 (only morphosyntactic rules mastered) was the lowest (.009). The proportions for patterns 010, 101 and 110 were all less than 0.02 . The marginal mastery proportion of morphosyntactic was .383 , of cohesive was .543 , and of lexical was .668 . This result indicated that the morphosyntactic attribute may be the most difficult attribute to be mastered and the lexical attribute may be the easiest.

Table 3 shows the estimated LCDM item parameters. From this table, we can recover the correct conditional item response probability for an attribute mastery pattern. For example, the 
correct item response probability of attribute mastery pattern 111 (pattern 8 ) for item 1 was calculated by $\omega_{81}=\exp (0.835+0.000+0.600+1.222) /(1+\exp (0.835+0.000+$ $0.600+1.222)) \approx 0.934$. Using such correct item response probabilities $\omega_{c i}$ along with attribute mastery pattern probabilities $\pi_{c}$, the conditional expectations and CSEMs in Table 4 and reliability indices in Table 5 were calculated.

Table 4 shows conditional expectations and CSEMs of sum- and sub-scores given each attribute mastery pattern. Table 4 also shows the unconditional expectations and standard errors of measurement of sum- and sub-scores. The range of expected sum scores of all mastery patterns was from 24.153 for pattern 111 (latent class 8) to 15.132 for pattern 000 (latent class 1). This indicates that even if examinees do not master any attributes, they are expected to correctly answer more than a half of all test items. In other words, this is a relatively easy test. In addition, the mean score difference between pattern 000 (latent class 1) and 111 (latent class 8) was about 9 points. CSEMs for the sum-score ranged from 1.769 to 2.470 and the difference was less than 1. Therefore, the sum-scores within an attribute mastery pattern vary by about 2 points. The conditional expectations and CSEM of total scores are shown in the Hasse diagram in Figure 1 to show the changes in expected scores. Expected score differences of more than two points were shown as black solid lines and differences less than two points were shown as grey dashed lines. This figure tells us possible learning paths and expected score changes with respect to the test. For example, mastering the lexical attribute can improve test scores by more than four points. Moreover, mastering the cohesive attribute improved scores by less than two points in this test. This conditional expectation graph can help teachers understand the relationship between skill mastery and improved test scores. Moreover, such visualized score differences may help to increase examinee motivation of learning. This is in contrast to only focusing on DCM-based attribute mastery where the resultant sum-score related to attribute mastery may be overlooked. We note that if attributes have a hierarchical relationship (e.g., Gierl, et al., 2009), the learning order of attribute may not be able to be changed. 
In the ECPE data, if an examinee masters the morphosyntactic attribute, then the expected sum-score was 23.828 (CSEM=1.830). Similarly, if an examinee does not master the lexical attribute, the expected sum-score becomes 15.399 (CSEM=2.466). In addition, the difference of expected sum-scores between mastery and non-mastery of each attribute was about 6 points. From these results, to get the highest score in the ECPE test, it is most effective to be a master of morphosyntactic rules.

The sub-score analysis also provided information about the ECPE test, overall. Mastering and not mastering the morphosyntactic attribute resulted in expected scores of 10.897 (CSEM = 1.291) and 6.699 (CSEM = 1.724), respectively. Similarly, the difference between expected subscores for masters and non-masters of the lexical rules attribute was 5.317 points. However, the expected sub-score of the cohesive rules attribute was 5.346 for mastery $(\mathrm{CSEM}=0.732)$ and 4.070 for non-mastery $(\mathrm{CSEM}=1.072)$ as there were six items measuring the cohesive rules attribute, a smaller number than the other attributes. This result also indicated that items measuring the cohesive rules attribute may not have large effects on the sub-score. Note that the sum-score difference between non-masters and masters was larger than the difference of subscores in the cohesive attribute. This is due to the correlation among attributes. As the three attributes were positively correlated with each other, if the cohesive attribute was not mastered by an examinee, it is likely that the other two attributes were also not mastered by the examinee. Therefore, the six-point difference of the sum-score was caused not only by non-masters the cohesive attribute but also by non-masters the morphosyntactic or lexical attributes even if the morphosyntactic or lexical attributes were marginalized.

Table 5 provides the results of observed score-based reliabilities and attribute reliabilities proposed by Templin and Bradshaw (2013). The reliabilities ranged from $.731\left(\rho_{\mathrm{DCM}}^{\left(\alpha_{2}\right)}\right)$ for the cohesive rules attribute sub-score to $.749\left(\rho_{\mathrm{DCM}}\right)$ for the sum score. This means that more than $73 \%$ of the variance of the sub-score was explained by the between-attribute mastery pattern average scores. The sub-score reliabilities were smaller than the sum score reliability due to the smaller number of items of the sub-scores. The values of sub-score reliabilities between 
attribute-specific and unconditional reliabilities were very similar. For example, the sub-score reliabilities of morphosyntactic was $\rho_{\mathrm{DCM}}^{(1)}=.654$ and $\rho_{\mathrm{DCM}}^{\left(1, \alpha_{1}\right)}=.645$. This result implied that attribute mastery and non-mastery may become a source of variance of sub-scores because the morphosyntactic attribute had a large impact on both sum and sub-scores. The sub-score reliabilities of the lexical rules attribute were $\rho_{\mathrm{DCM}}^{(3)}=.709$ and $\rho_{\mathrm{DCM}}^{\left(3, \alpha_{3}\right)}=.699$ and were a little larger than $\rho_{\mathrm{DCM}}^{(1)}$ or $\rho_{\mathrm{DCM}}^{\left(1, \alpha_{1}\right)}$. Therefore, the lexical rules attribute also had a large impact on the sum score. The sub-score reliabilities of the cohesive rules attribute were $\rho_{\mathrm{DCM}}^{(2)}=.334$ and $\rho_{\mathrm{DCM}}^{\left(2, \alpha_{2}\right)}=.331$. These values were much smaller than the other reliabilities. The cohesive rules reliabilities indicated that the within-pattern error variance was greater than the between-pattern variance in the sub-score of the lexical rules attribute. In the case of a test or sub-score with small number of items that are either very easy or difficult for examinees, the score may have a floor or ceiling effect, reducing its variance. Such small variances can cause low reliabilities so increasing the number of items measuring the cohesive attribute can improve the reliability. Attribute reliabilities from Templin and Bradshaw (2013) showed higher values than observed sub-score reliabilities. The order of three attribute reliabilities were the same as the sub-score reliabilities: the lexical rules attribute had the highest reliability and the cohesive rules attribute had the lowest. The absolute values between two types of reliabilities were different because the two reliabilities reflect different aspects of their respective methods. Templin and Bradshaw's reliabilities are based on test-retest reliability. In other words, it is an estimate of the consistency of attribute mastery between two parallel tests. On the other hand, our sub-score reliabilities are the ratio of the variance accounted for by attribute mastery. From both types of reliabilities, the lexical rules attribute had a high value whose attribute reliability was 0.919 , indicating the attribute was satisfactorily measured by the test. The morphosyntactic rules attribute had high attribute reliability $(=0.891)$ but not sub-score reliability. This might indicate an area where the ECPE needs some improvement. The attribute reliability of the cohesive rules attribute was moderate $(=0.802)$ in but the sub-score reliability was small. This result indicates 
that if the test developer needs to improve the cohesive rules attribute diagnoses, it may necessitate the development of new items measuring the cohesive rules attribute.

In summary, from perspective of the proposed observed reliability, the morphosyntactic rules attribute and the lexical rules attribute were sources of test score variance and the cohesive rules attribute was not a source of test score variance. These results provide several suggestions about where one could improve the ECPE. Specifically, the cohesive rules attribute had only a relatively small effect on the sum score and sub-score variance indicating cohesive rules items should be added. Alternatively, omitting the attribute from the test would show little impact on the variance of the test score and would shorten the test.

\section{Simulation Study}

\section{Simulation Settings}

We present results of a simulation study designed to compare the nature of our proposed

observed sub-score-based reliability indices, especially $\rho_{\mathrm{DCM}}^{\left(a, \alpha_{a}\right)}$, to the attribute reliability index of Templin and Bradshaw (2013). In addition, we assessed the correlation between the two types of reliabilities and the correct attribute recovery rate. This simulation provides an insight into the connection of the observed sub-score-based reliability index to the latent attribute reliability. We also examined the effect of the test length on the both sub-score-based and attribute reliabilities.

In the first setting, we manipulated the number of items measuring only one attribute and examined the effect of item size per items. The Q-matrices employed in this simulation are shown in Table 6. The number of attributes was fixed to three for all conditions. The smallest item condition (named Q1) had nine Q1 basic items, as shown in Table 6. The Q2 condition items consisted of Q1 basic items plus two Q2 additional items that measured only the first attribute for a total of 11 items. Similarly, the Q3 condition had Q1 basic items and Q3 additional items which contained two items measuring the first attribute and the other two measuring the second attribute. Finally, the Q4 condition used the Q1 basic items and Q4 items that consisted of double the amount of the Q3 additional items. We used these additional items to evaluate the effect of the increase of the number of items measuring attributes. Four Q-matrices were used in 
this simulation. Note that the items measuring the third attribute ware not added. We expected the reliability related to the third attribute to not be improved in this simulation. In other words, we held the number of items measuring the third attribute constant as a control to compare with as we expected the reliability of an attribute to be related to the number of items measuring that attribute. In the second setting, we also set the other items condition to investigate the effect of the test length on reliability. The first condition had Q1 basic items in Table 6, which is

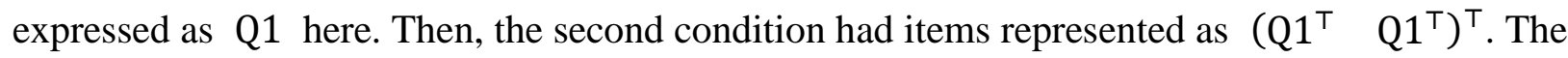
third and fourth conditions assumed stacked three and four Q1s.

We conducted 100 replications of our simulation. For each replication, the true LCDM parameters were generated as follows. The intercepts $\lambda_{0}$ were generated from Uniform $(-2,-1)$, the main effect for the first attribute was generated from Uniform $(1.5,2.5)$, the second attribute main effects were generated from Uniform $(2,3)$, and the third attribute main effects were generated from Uniform(2.5, 3.5). Here, the items measuring the first attribute had relatively low discrimination values and the items measuring the third attribute had the highest discrimination values. In addition, all first-order interactions were sampled from Uniform $(1,2)$ to satisfy the monotonicity constraint and to represent attributes with small interaction parameters. From the ECPE data analysis, the interaction parameter estimates were not large so we mimicked such moderate values in this setting. The sample size was 1,000 per replication. Attribute patterns were generated with bivariate tetrachoric correlations of .4 and with proportions of masters set to 0.5 . Mplus software was used to estimate parameters. Non converged model runs were excluded from final analysis. The $\rho_{\mathrm{DCM}}^{\left(a, \alpha_{a}\right)}$, attribute reliability by Templin and Bradshaw, and correct attribute recoveries that were based on the rounded expected-a-posteriori values were calculated with the estimated parameters.

\section{Results}

Table 7 shows the means and SDs of sub-score and attribute reliabilities and the attribute recovery rate for each attribute. The Q1 condition showed the lowest sub-score reliabilities. As we expected, the observed reliability for the first attribute gradually improved from conditions 
Q2 to Q4. This tendency was also observed for the sub-score for the second attribute. The value of sub-score reliabilities for the second attribute in Q1 and Q2 were almost the same 0.674 or 0.673 but became 0.673 and 0.740 in the Q3 and Q4 conditions. Finally, the sub-score reliability of the third attribute was roughly equal across all four Q-matrix conditions. The attribute reliabilities and attribute recovery rates showed the same tendency of sub-score reliabilities. In other words, as more items were added, the values of each respective statistic increased. However, sub-score reliabilities showed smaller values than the attribute reliabilities or attribute recovery rates.

Table 8 shows the correlations among sub-score reliabilities, attribute reliabilities, and attribute recovery rates for each condition. The correlation between sub-score and attribute reliabilities were higher than 0.9 for the Q2 to Q4 conditions. Even in the Q1 condition, the correlations for the second and third attributes were close to 0.90 but the correlation for the first attribute was about 0.8 , which was the lowest. The effects of adding items were not clear in this simulation but the results indicated the minimum items condition (Q1) might not have enough items to provide high correlations. On the other hand, correlations between the two types of reliabilities and attribute recovery rates had relatively lower values and did not exceed 0.9 . The effects of adding items were limited again. In summary, the sub-score- and attribute-based reliability were linearly related, but they indicated different aspects of attribute recovery.

The result of the second simulation that assessed the effect of the number of items on the two types of reliabilities was shown in Figure 2. The figure clearly shows that the increase in the number of items was related to the increase of both sub-score and attribute reliabilities. In other words, the two reliabilities were monotonically increasing functions of test length. However, the absolute values between two reliabilities were different. The sub-score-based reliabilities were smaller than the attribute reliabilities. This result indicated that even if the observed score reliabilities are low, attribute reliability can be larger. 


\section{Discussion}

Observed test scores are typically not a focus in DCMs but are still meaningful for teachers or students who use tests formatively. This study derived conditional sum- and subscore distributions of DCMs and introduced observed score-based reliability indices to improve the usefulness of test scores for assessments analyzed with DCMs. In addition, we analyzed the ECPE data from the perspective of its observed scores. The results showed that the cohesive rules attribute had a smaller effect on the test score than the other two attributes. A small simulation study demonstrated adding items increased the sub-score reliabilities. Moreover, the simulation study revealed the similarity between sub-score reliability and a previously developed index of attribute reliability.

The purpose of our observed score-related metrics was to improve the usefulness of simple sum-scores that are easy to use for educational practitioners and that help test developers understand test behavior in an intuitive way. Conditional expectations can be used to construct a test to have a desired average score and help to make optimal strategies for skill acquisition and teaching using an expected score gain. Observed score reliabilities could provide such a perspective to improve the test. The simulation study indicated that improving sub-score reliability by adding items also increases the reliability of other attributes. Our ECPE data analyses and our simulation demonstrated how to use our developed indices. From the simulation study, the observed attribute reliabilities were correlated, and the two reliabilities were similar. Our new observed score-based metrics are not just for quantifying the reliability of latent attributes but also for improving the understanding of a test.

We believe that the derived distributions of tests scores and reliability indices are best employed for understanding the nature of the diagnostic tests rather than individual test scores. This is because the main outcomes of DCMs are attribute mastery patterns or mastery probabilities. The derived indices give hints on how to improve a test or provide a viewpoint to understand the test itself. The diagnostic results with DCMs necessitate the use of a reliable test and the derived indices can contribute to the improvement of test quality in an intuitive way. Our 
indices are easy to use, which may be beneficial for practitioners. The methods shown in this paper need only the estimated item parameters and mixing proportion parameters and do not need complex computations for calculation of the reliability indices. In fact, we can calculate various expectations and variances based on the parameter tables in published papers. Further, although we conditioned on a single attribute in our paper, one can also derive other conditional distributions of test scores. For example, one can calculate expectations and variances conditional on two or more attributes to assess the interaction effects between attributes. Observed score-based reliability indices for attribute interactions can be also defined in similar ways proposed in this article.

The empirical data example showed which attribute had relatively large effects on the sum or sub-scores. The original item parameter estimates were also important, but it was not clear what their effects on the test score were. The proposed reliability indices can be used to refine a test because they represent the variance components explained by the attributes. Therefore, they indicate what kind of items should be added to a test or omitted from a test. In addition, the conditional distribution results can be used to understand which attribute mastery pattern is expected to score high or low on the test. If the specific pattern gained unexpectedly high or low scores, it may indicate a misspecification issue with the Q-matrix. The proposed indices and conditional distributions can reveal the effect of latent attributes on the test score more directly than previous indices. As a final note, we do not suggest that the sum and subscores should be employed for diagnoses. Rather, these scores can be used to understand diagnostic results based on statistical estimation of results from specific data.

Our simulation showed a relationship between sub-score reliabilities and attribute reliabilities from a previously developed DCM reliability index. This fact implies our observed reliability indies shared some properties to previously developed latent attribute reliability indices. Moreover, our simulation study implied if test developers want to improve attribute reliability in a test, an easy way is to add items to increase observed score reliability. In other words, an increase the observed score reliability also results in an increase of attribute 
classification reliability. The observed score reliability can be an easy-to-calculate alternative of the attribute reliability index and can provide an intuitive alternative perspective of attribute reliability.

We also mention a thought about the discrepancy in values between our observed score reliability indices and the attribute reliabilities from Templin and Bradshaw (2013). One interpretation can be thought to depend on the purpose and situation for using an observed test. As with all reliability indices, it is difficult to define a criterion that is applicable for all situations. If we think about a conservative criterion, this means that the lowest values of reliabilities must be improved. On the other hand, an optimistic perspective indicates that it is enough to satisfy some reliabilities based on some prespecified criterion. However, there are not stringent rules to decide such a perspective so caution in interpretation is advised.

Future research could pursue investigating more detailed mathematical properties of observed score reliabilities and the relationship between the two types of reliabilities. Another direction for future research would be comparing other previously developed indices and our observed score index. This direction would also be meaningful to further understanding our observed score-based reliabilities. However, final decisions about a test should be carefully made by using multiple indices as our proposed indices represent one aspect of the nature of attributes. We also must note that model selection is needed before calculating the proposed indices as the proposed indices depend on estimates of item parameters and mixing parameters in selected DCMs. Additionally, variability of parameter estimates has the potential to affect the values of the proposed indices. Another direction for future research is the development of standard error estimates for the proposed observed score reliability indices.

\section{References}

American Educational Research Association, American Psychological Association, \& National Council on Measurement in Education. (2014). Standards for educational and psychological testing. Washington, DC: American Educational Research Association 
Chen, Y., Liu, Y., \& Xu, S. (2018). Mutual information reliability for latent class analysis. Applied Psychological Measurement, 42, 460-477. https://doi.org/10.1177/0146621617748324

Cui, Y., Gierl, M. J., \& Chang, H. H. (2012). Estimating classification consistency and accuracy for cognitive diagnostic assessment. Journal of Educational Measurement, 49, 19-38. https://doi.org/10.1111/j.1745-3984.2011.00158.x

de la Torre, J. (2008). An empirically based method of Q-matrix validation for the DINA model: Development and applications. Journal of Educational Measurement, 45, 343-362. http://dx.doi.org/10.1111/j.1745-3984.2008.00069.x

de la Torre, J. (2011). The generalized DINA model framework. Psychometrika, 76, 179-199. https://doi.org/10.1007/S11336-011-9207-7

de la Torre, J., \& Akbay, L. (2019). Implementation of cognitive diagnosis modeling using the GDINA R package. Eurasian Journal of Educational Research, 19, 171-192. https://doi.org/10.14689/ejer.2019.80.9

DiBello, L. V., Roussos, L. A., \& Stout, W. (2006). Review of cognitively diagnostic assessment and a summary of psychometric models. In C. R. Rao \& S. Sinharay (Eds.), Handbook of Statistics (Vol. 26, pp. 979-1030). Amsterdam: Elsevier. https://doi.org/10.1016/S01697161(06)26031-0

Feinberg, R. A., \& Jurich, D. P. (2017). Guidelines for interpreting and reporting subscores. Educational Measurement: Issues and Practice, 36, 5-13. https://doi.org/10.1111/emip.12142

Feldt, L. S., Steffen, M., \& Gupta, N. C. (1985). Comparison of five methods for measurement at specific score levels. Applied Psychological Measurement, 9, 351-361. https://doi.org/10.1177/014662168500900402

George, A. C., Robitzsch, A., Kiefer, T., Groß, J., \& Ünlü, A. (2016). The R package CDM for cognitive diagnosis models. Journal of Statistical Software, 74. https://doi.org/10.18637/jss.v074.i02 
Gierl, M. J., Cui, Y., \& Zhou, J. (2009). Reliability and attribute-based scoring in cognitive diagnostic assessment. Journal of Educational Measurement, 46, 293-313. https://doi.org/10.1111/j.1745-3984.2009.00082.x

Haberman, S. J. (2008). When can subscores have value? Journal of Educational and Behavioral Statistics, 33, 204-229. https://doi.org/10.3102/1076998607302636

Henson, R., DiBello, L., \& Stout, B. (2018). A generalized approach to defining item discrimination for DCMs. Measurement: Interdisciplinary Research and Perspectives, 16(1), 18-29. http://dx.doi.org/10.1080/15366367.2018.143685

Henson, R. A., Templin, J. L., \& Willse, J. T. (2009). Defining a family of cognitive diagnosis models using log-linear models with latent variables. Psychometrika, 74, 191-210. https://doi.org/10.1007/S11336-008

Henson, R., Templin, J., \& Douglas, J. (2007). Using efficient model based sum-scores for conducting skills diagnoses. Journal of Educational Measurement, 44, 361-376. https://doi.org/10.1111/j.1745-3984.2007.00044.x

Johnson, M. S., \& Sinharay, S. (2018). Measures of agreement to assess attribute-level classification accuracy and consistency for cognitive diagnostic assessments. Journal OfEducational Measurement, 55, 635-664. https://doi.org/10.1111/jedm.12196

Johnson, M. S., \& Sinharay, S. (2019). The reliability of the posterior probability of skill attainment in diagnostic classification models. Journal of Educational and Behavioral Statistics, $X X(X), 1-27$. https://doi.org/10.3102/1076998619864550

Lai, H., Gierl, M. J., \& Babenko, O. (2015). Application of conditional means for diagnostic scoring. International Journal of Learning, Teaching and Educational Research, 12, 61-79.

Leighton, J. P., \& Gierl, M. J. (Eds.). (2007). Cognitive diagnostic assessment for education: Theory and applications. New York, NY: Cambridge University Press.

Liu, R., Huggins-Manley, A. C., \& Bulut, O. (2018). Retrofitting diagnostic classification models to responses from IRT-based assessment forms. Educational and Psychological Measurement, 78, 357-383. https://doi.org/10.1177/0013164416685599 
Liu, R., Qian, H., Luo, X., \& Woo, A. (2018). Relative diagnostic profile: A subscore reporting framework. Educational and Psychological Measurement, 78, 1072-1088. https://doi.org/10.1177/0013164417740170

Ma, W., \& de la Torre, J. (2017). GDINA: The generalized DINA model framework. Retrieved from https://cran.r-project.org/package=GDINA

Muthén, L. K., \& Muthén, B. O. (1998-2017). Mplus User’s Guide. Eighth Edition. Los Angeles, CA: Muthén \& Muthén.

Ravand, H. (2015). Cognitive diagnostic modeling using R. Practical Assessment, Research \& Evaluation, 20, 1-12.

Ravand, H., \& Baghaei, P. (2019). Diagnostic classification models: Recent developments, practical issues, and prospects. International Journal of Testing, 1-33. https://doi.org/10.1080/15305058.2019.1588278

Rupp, A. A., \& Templin, J. (2008). Unique characteristics of diagnostic classification models: A comprehensive review of the current state-of-the-art. Measurement: Interdisciplinary Research \& Perspective, 6, 219-262. https://doi.org/10.1080/15366360802490866

Rupp, A. A., Templin, J. L., \& Henson, R. A. (2010). Diagnostic measurement: Theory, methods and applications. New York, NY: Guilford Press.

Sessoms, J., \& Henson, R. A. (2018). Applications of diagnostic classification models: A literature review and critical commentary. Measurement: Interdisciplinary Research and Perspectives, 16, 1-17. https://doi.org/10.1080/15366367.2018.1435104

Sinharay, S. (2010). How often do subscores have added value? Results from operational and simulated data. Journal of Educational Measurement, 47, 150-174. https://doi.org/10.1111/j.1745-3984.2010.00106.x

Sinharay, S., Puhan, G., \& Haberman, S. J. (2010). Reporting diagnostic scores in educational testing: Temptations, Pitfalls, and some solutions. Multivariate Behavioral Research, 45, 553-573. https://doi.org/10.1080/00273171.2010.483382 
Tatsuoka, K. K. (1983). Rule space: An approach for dealing with misconceptions based on item response theory. Jounal of Educational Measurement, 20, 345-354. https://doi.org/10.1111/j.1745-3984.1983.tb00212.x

Templin, J., \& Bradshaw, L. (2013). Measuring the reliability of diagnostic classification model examinee estimates. Journal of Classification, 30, 251-275. https://doi.org/10.1007/s00357013-9129-4

Templin, J., \& Bradshaw, L. (2014). Hierarchical diagnostic classification models: A family of models for estimating and testing attribute hierarchies. Psychometrika, 79, 317-339. https://doi.org/10.1007/s11336-013-9362-0

Templin, J., \& Hoffman, L. (2013). Obtaining diagnostic classification model estimates using Mplus. Educational Measurement: Issues and Practice, 32, 37-50. https://doi.org/https://doi.org/10.1111/emip.12010

von Davier, M. (2008). A general diagnostic model applied to language testing data. The British Journal of Mathematical and Statistical Psychology, 61, 287-307. https://doi.org/10.1348/000711007X193957

Wang, W., Song, L., Chen, P., Meng, Y., \& Ding, S. (2015). Attribute-level and pattern-level classification consistency and accuracy indices for cognitive diagnostic assessment. Journal of Educational Measurement, 52, 457-476. https://doi.org/10.1111/jedm.12096

Yao, L. (2010). Reporting valid and reliable overall scores and domain scores. Journal of Educational Measurement, 47, 339-360. https://doi.org/10.1111/j.1745-3984.2010.00117.x 
Table 1.

$Q$ matrix of ECPE data

\begin{tabular}{|c|c|c|c|c|}
\hline \# of Item & $\begin{array}{c}\text { Morphosyntactic } \\
\text { Attribute }\end{array}$ & $\begin{array}{l}\text { Cohesive } \\
\text { Attribute }\end{array}$ & $\begin{array}{l}\text { Lexical } \\
\text { Attribute }\end{array}$ & Sum \\
\hline 1 & 1 & 1 & 0 & 2 \\
\hline 2 & 0 & 1 & 0 & 1 \\
\hline 3 & 1 & 0 & 1 & 2 \\
\hline 4 & 0 & 0 & 1 & 1 \\
\hline 5 & 0 & 0 & 1 & 1 \\
\hline 6 & 0 & 0 & 1 & 1 \\
\hline 7 & 1 & 0 & 1 & 2 \\
\hline 8 & 0 & 1 & 0 & 1 \\
\hline 9 & 0 & 0 & 1 & 1 \\
\hline 10 & 1 & 0 & 0 & 1 \\
\hline 11 & 1 & 0 & 1 & 2 \\
\hline 12 & 1 & 0 & 1 & 2 \\
\hline 13 & 1 & 0 & 0 & 1 \\
\hline 14 & 1 & 0 & 0 & 1 \\
\hline 15 & 0 & 0 & 1 & 1 \\
\hline 16 & 1 & 0 & 1 & 2 \\
\hline 17 & 0 & 1 & 1 & 2 \\
\hline 18 & 0 & 0 & 1 & 1 \\
\hline 19 & 0 & 0 & 1 & 1 \\
\hline 20 & 1 & 0 & 1 & 2 \\
\hline 21 & 1 & 0 & 1 & 2 \\
\hline 22 & 0 & 0 & 1 & 1 \\
\hline 23 & 0 & 1 & 0 & 1 \\
\hline 24 & 0 & 1 & 0 & 1 \\
\hline 25 & 1 & 0 & 0 & 1 \\
\hline 26 & 0 & 0 & 1 & 1 \\
\hline 27 & 1 & 0 & 0 & 1 \\
\hline 28 & 0 & 0 & 1 & 1 \\
\hline Sum & 13 & 6 & 18 & \\
\hline
\end{tabular}


Table 2.

Attribute mastery pattern combination and estimated proportion of each pattern, mastery and non-mastery

\begin{tabular}{ccccc}
\hline $\begin{array}{c}\text { Latent Class } \\
c\end{array}$ & $\begin{array}{c}\text { Morphosyntactic } \\
\text { Attribute }\end{array}$ & $\begin{array}{c}\text { Cohesive } \\
\text { Attribute }\end{array}$ & $\begin{array}{c}\text { Lexical } \\
\text { Attribute }\end{array}$ & $\pi_{c}$ \\
\hline 1 & 0 & 0 & 0 & .301 \\
2 & 0 & 0 & 1 & .129 \\
3 & 0 & 1 & 0 & .012 \\
4 & 0 & 1 & 1 & .175 \\
5 & 1 & 0 & 0 & .009 \\
6 & 1 & 0 & 1 & .018 \\
7 & 1 & 1 & 0 & .011 \\
8 & 1 & 1 & 1 & .346 \\
\hline Master & .383 & .543 & .668 & \\
Non-masters & .617 & .457 & .332 & \\
\hline
\end{tabular}

Note: the bottom two rows list the marginal proportion of masters and non-masters for each attribute. 
Table 3.

Estimated item parameters of LCDM

\begin{tabular}{|c|c|c|c|c|c|c|c|}
\hline Item & $\lambda_{i, 0}$ & $\lambda_{i, 1,(1)}$ & $\lambda_{i, 1,(2)}$ & $\lambda_{i, 1,(3)}$ & $\lambda_{i, 2,(1,2)}$ & $\lambda_{i, 2,(1,3)}$ & $\lambda_{i, 2,(2,3)}$ \\
\hline 1 & 0.835 & 0.000 & 0.600 & & 1.222 & & \\
\hline 2 & 1.037 & & 1.247 & & & & \\
\hline 3 & -0.340 & 0.748 & & 0.346 & & 0.535 & \\
\hline 4 & -0.139 & & & 1.691 & & & \\
\hline 5 & 1.082 & & & 2.015 & & & \\
\hline 6 & 0.865 & & & 1.692 & & & \\
\hline 7 & -0.106 & 2.855 & & 0.952 & & -0.952 & \\
\hline 8 & 1.482 & & 1.922 & & & & \\
\hline 9 & 0.119 & & & 1.195 & & & \\
\hline 10 & 0.055 & 2.050 & & & & & \\
\hline 11 & -0.039 & 0.818 & & 0.961 & & 0.777 & \\
\hline 12 & -1.769 & 0.000 & & 1.290 & & 1.515 & \\
\hline 13 & 0.660 & 1.630 & & & & & \\
\hline 14 & 0.176 & 1.368 & & & & & \\
\hline 15 & 0.996 & & & 2.114 & & & \\
\hline 16 & -0.104 & 2.341 & & 0.892 & & -0.864 & \\
\hline 17 & 1.354 & & 0.767 & 0.596 & & & 0.076 \\
\hline 18 & 0.926 & 1.389 & & & & & \\
\hline 19 & -0.195 & 1.848 & & & & & \\
\hline 20 & -1.389 & 0.243 & & 0.908 & & 1.410 & \\
\hline 21 & 0.164 & 1.053 & & 1.130 & & 0.042 & \\
\hline 22 & -0.872 & & & 2.245 & & & \\
\hline 23 & 0.664 & & 2.071 & & & & \\
\hline 24 & -0.673 & & 1.522 & & & & \\
\hline 25 & 0.092 & 1.136 & & & & & \\
\hline 26 & 0.164 & & & 1.119 & & & \\
\hline 27 & -0.887 & 1.713 & & & & & \\
\hline 28 & 0.568 & & & 1.745 & & & \\
\hline
\end{tabular}

Note. $N=2922$ and this is a part of Table 1 of Templin and Hoffman (2013). 
Table 4.

Conditional sum- and sub-scores expectation and standard error of measurement of LCDM

\begin{tabular}{|c|c|c|c|c|c|c|c|c|}
\hline \multirow{3}{*}{ Latent Class } & \multirow{2}{*}{\multicolumn{2}{|c|}{ Sum-score }} & \multicolumn{6}{|c|}{ Sub-score } \\
\hline & & & \multicolumn{2}{|c|}{ Morphosyntactic } & \multicolumn{2}{|c|}{ Cohesive } & \multicolumn{2}{|c|}{ Lexical } \\
\hline & Expecatation & CSEM & Expecatation & CSEM & Expecatation & CSEM & Expecatation & CSEM \\
\hline 000 & 15.132 & 2.470 & 5.969 & 1.707 & 4.044 & 1.080 & 9.352 & 1.979 \\
\hline 001 & 19.505 & 2.214 & 7.382 & 1.706 & 4.124 & 1.055 & 13.724 & 1.649 \\
\hline 010 & 16.304 & 2.359 & 6.079 & 1.691 & 5.216 & 0.796 & 9.450 & 1.962 \\
\hline 011 & 20.646 & 2.093 & 7.493 & 1.689 & 5.265 & 0.769 & 13.791 & 1.632 \\
\hline 100 & 18.242 & 2.278 & 9.078 & 1.416 & 4.044 & 1.080 & 10.900 & 1.864 \\
\hline 101 & 22.885 & 1.935 & 10.763 & 1.325 & 4.124 & 1.055 & 15.544 & 1.425 \\
\hline 110 & 19.541 & 2.136 & 9.315 & 1.362 & 5.342 & 0.734 & 10.998 & 1.846 \\
\hline 111 & 24.153 & 1.769 & 11.000 & 1.267 & 5.392 & 0.705 & 15.610 & 1.406 \\
\hline$\alpha_{1}$ master & 23.828 & 1.830 & 10.897 & 1.291 & & & & \\
\hline$\alpha_{1}$ non-master & 17.635 & 2.391 & 6.699 & 1.724 & & & & \\
\hline$\alpha_{2}$ master & 22.759 & 1.978 & & & 5.346 & 0.732 & & \\
\hline$\alpha_{2}$ non-master & 16.735 & 2.447 & & & 4.070 & 1.072 & & \\
\hline$\alpha_{3}$ master & 22.301 & 2.031 & & & & & 14.767 & 1.559 \\
\hline$\alpha_{3}$ non-master & 15.399 & 2.466 & & & & & 9.450 & 1.979 \\
\hline Unconditional & 20.008 & 4.252 & 8.308 & 2.640 & 4.763 & 1.105 & 13.001 & 3.117 \\
\hline
\end{tabular}

Note. CSEMs are conditional standard error of measurement 
Table 5.

Observed score-based reliability and attribute reliability by Templin and Bradshaw (2013) of LCDM

\begin{tabular}{ccccc}
\hline \multirow{2}{*}{ Reliability type } & Sum-score & \multicolumn{4}{c}{ Sub-score } \\
\cline { 3 - 5 } & & Morphsyntactic & Cohesive & Lexical \\
\hline Unconditional & .749 & .654 & .334 & .709 \\
Attribute 1 & & & & \\
(Morphsyntactic) & .734 & .645 & .331 & \\
Attribute 2 & & & & .699 \\
$\begin{array}{c}\text { (Cohesive) } \\
\text { Attribute 3 }\end{array}$ & .731 & & & \\
(Lexical) & .736 & & & \\
\hline Attribute & & & & \\
reliability & & & & \\
\hline
\end{tabular}


Table 6.

Basic Q-matrix and additional items for simulation study

\begin{tabular}{|c|c|c|c|}
\hline \multirow{2}{*}{ Condition } & \multicolumn{3}{|c|}{ Attribute } \\
\hline & 1 & 2 & 3 \\
\hline \multirow{9}{*}{ Q1 basic items } & 1 & 0 & 0 \\
\hline & 0 & 1 & 0 \\
\hline & 0 & 0 & 1 \\
\hline & 1 & 0 & 0 \\
\hline & 0 & 1 & 0 \\
\hline & 0 & 0 & 1 \\
\hline & 1 & 1 & 0 \\
\hline & 1 & 0 & 1 \\
\hline & 0 & 1 & 1 \\
\hline Q2 additional & 1 & 0 & 0 \\
\hline items & 1 & 0 & 0 \\
\hline \multirow{4}{*}{$\begin{array}{l}\text { Q3 additional } \\
\text { items }\end{array}$} & 1 & 0 & 0 \\
\hline & 1 & 0 & 0 \\
\hline & 0 & 1 & 0 \\
\hline & 0 & 1 & 0 \\
\hline \multirow{8}{*}{$\begin{array}{c}\text { Q4 additional } \\
\text { items }\end{array}$} & 1 & 0 & 0 \\
\hline & 1 & 0 & 0 \\
\hline & 0 & 1 & 0 \\
\hline & 0 & 1 & 0 \\
\hline & 1 & 0 & 0 \\
\hline & 1 & 0 & 0 \\
\hline & 0 & 1 & 0 \\
\hline & 0 & 1 & 0 \\
\hline
\end{tabular}


Table 7.

Means and SDs of observed score reliabilities, attribute reliabilities, and attribute recovery rates over 100 replications for each Q-matrix condition

\begin{tabular}{|c|c|c|c|c|c|c|c|c|c|c|c|c|c|}
\hline \multirow{3}{*}{$\begin{array}{l}\text { Q-matrix } \\
\text { condition }\end{array}$} & \multirow{3}{*}{ Statics } & \multicolumn{12}{|c|}{ Attribute } \\
\hline & & \multicolumn{4}{|c|}{1} & \multicolumn{4}{|c|}{2} & \multicolumn{4}{|c|}{3} \\
\hline & & Mean & ( & $\mathrm{SD}$ & ) & Mean & ( & $\mathrm{SD}$ & ) & Mean & ( & SD & ) \\
\hline \multirow{3}{*}{ Q1 } & Sub-score reliability & .593 & ( & .033 & ) & .674 & ( & .028 & ) & .732 & ( & .021 & ) \\
\hline & Attribute reliability & .763 & ( & .045 & ） & .873 & ( & .027 & ) & .932 & ( & .017 & ） \\
\hline & Attribute recovery rate & .827 & ( & .019 & ) & .882 & ( & .021 & ) & .922 & ( & .015 & ) \\
\hline \multirow{3}{*}{ Q2 } & Sub-score reliability & .641 & ( & .029 & ) & .673 & ( & .030 & ) & .734 & ( & .022 & ) \\
\hline & Attribute reliability & .861 & ( & .030 & ） & .871 & ( & .031 & ) & .935 & ( & .017 & ） \\
\hline & Attribute recovery rate & .876 & ( & .015 & ) & .884 & ( & .017 & ) & .926 & ( & .015 & ) \\
\hline \multirow{3}{*}{ Q3 } & Sub-score reliability & .649 & ( & .032 & ) & .740 & ( & .026 & ) & .730 & ( & .022 & ) \\
\hline & Attribute reliability & .870 & ( & .033 & ） & .949 & ( & .016 & ) & .931 & ( & .018 & ） \\
\hline & Attribute recovery rate & .880 & ( & .017 & ) & .927 & ( & .014 & ) & .924 & ( & .013 & ) \\
\hline \multirow{3}{*}{ Q4 } & Sub-score reliability & .693 & ( & .027 & ) & .783 & ( & .017 & ) & .734 & ( & .022 & ) \\
\hline & Attribute reliability & .924 & ( & .022 & ) & .979 & 1 & .006 & ) & .935 & ( & .016 & ) \\
\hline & Attribute recovery rate & .912 & ( & .015 & ) & .952 & ( & .011 & ) & .923 & ( & .013 & ) \\
\hline
\end{tabular}

Note. Attribute reliabilities were based on Templin and Bradshaw (2013). 
Table 8.

Correlations among sub-score reliabilities, attribute reliabilities, and attribute recovery rates over 100 replications for each Q-matrix condition

\begin{tabular}{cccccc}
\hline \multirow{2}{*}{$\begin{array}{c}\text { Q-matrix } \\
\text { condition }\end{array}$} & \multicolumn{2}{c}{ Combination } & \multicolumn{3}{c}{ Attribute } \\
\cline { 5 - 6 } Q1 & Sub-score reliability & Attribute reliability & .805 & .892 & .895 \\
& Sub-score reliability & Attribute recovery rate & .651 & .777 & .633 \\
& Attribute reliability & Attribute recovery rate & .693 & .823 & .687 \\
\hline \hline \multirow{2}{*}{ Q2 } & Sub-score reliability & Attribute reliability & .942 & .934 & .914 \\
& Sub-score reliability & Attribute recovery rate & .719 & .666 & .703 \\
\hline \hline & Attribute reliability & Attribute recovery rate & .745 & .732 & .714 \\
\hline \multirow{2}{*}{ Q3 } & Sub-score reliability & Attribute reliability & .930 & .952 & .924 \\
& Sub-score reliability & Attribute recovery rate & .719 & .820 & .565 \\
& Attribute reliability & Attribute recovery rate & .756 & .829 & .620 \\
\hline \hline
\end{tabular}

Note. Attribute reliabilities were based on Templin and Bradshaw (2013). 
Figure 1.

Hasse diagram of attribute mastery patterns and conditional expectations and standard error of measurements

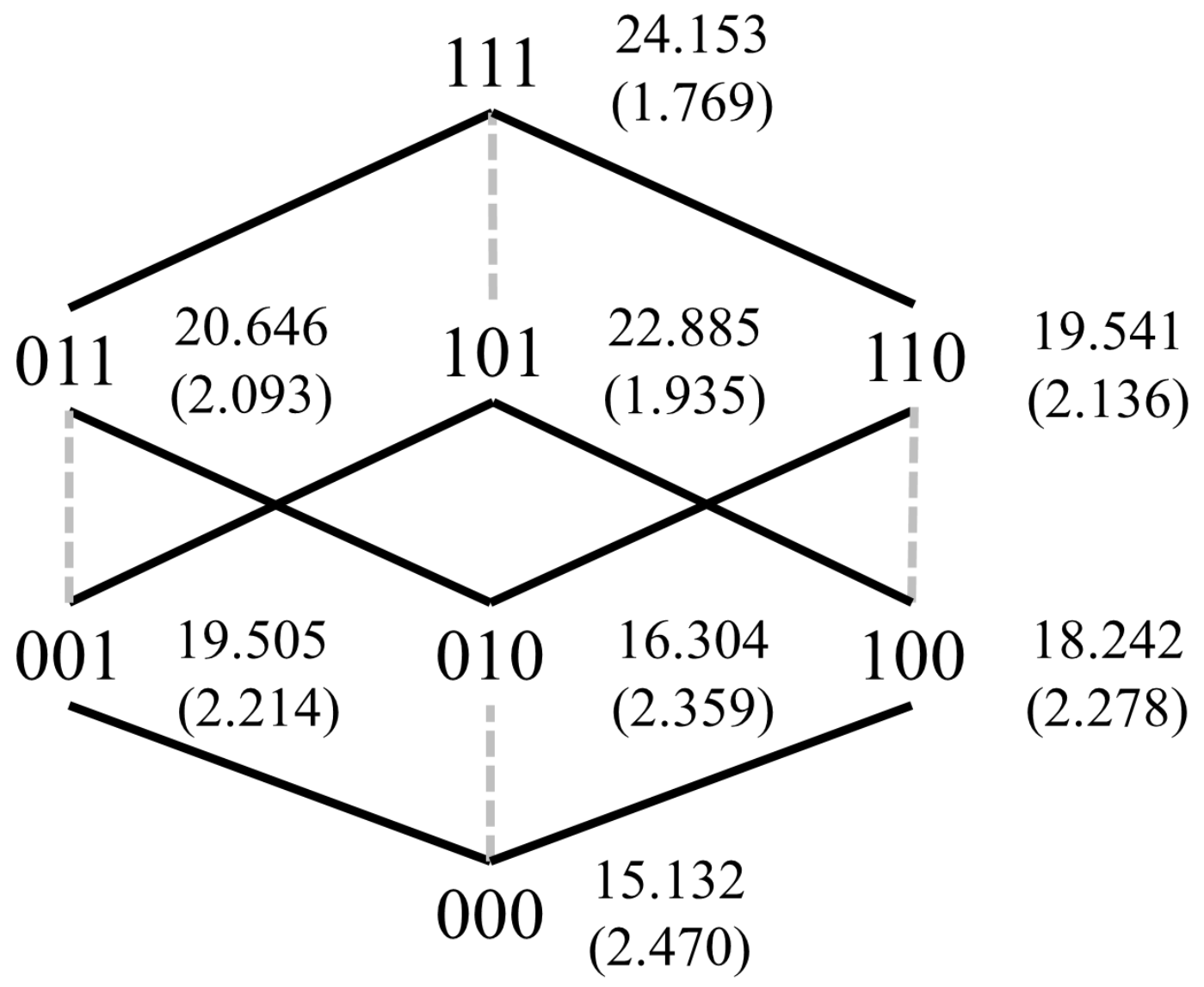

Note. Conditional standard errors of measurement were shown in parentheses, the black solid lines represented the conditional expectations differences were more than two points, and the grayed dashed lines indicated less than two points. 
Figure 2.

The test length effect on sub-score-based and attribute reliabilities

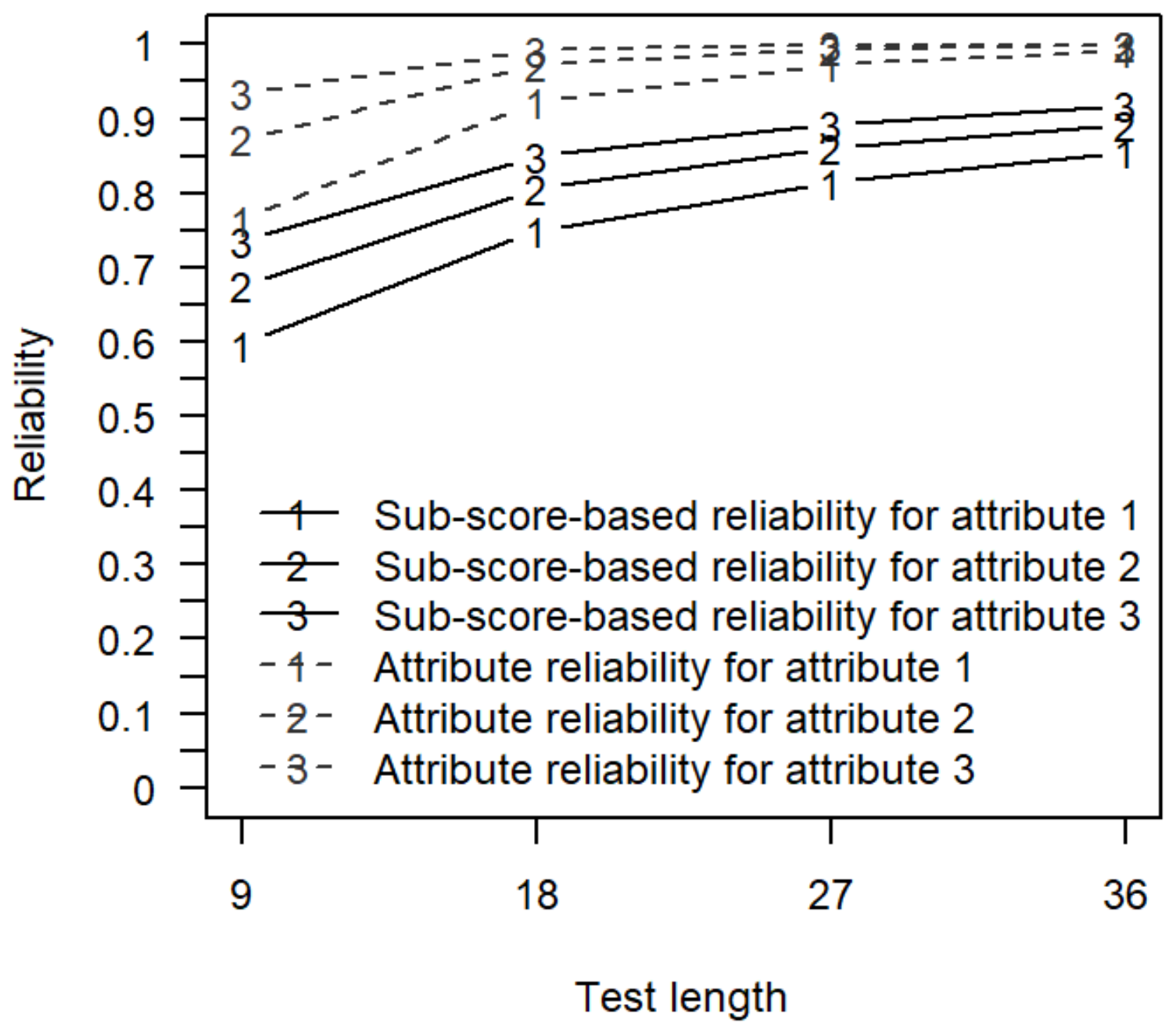

